\title{
MS09-05 | Molecular Replacement Model Preparation and its Automation in
}

\section{MoRDa ANd MrBUMP}

Lebedev, Andrey (STFC-UKRI, Didcot, GBR); Vagin, Alexy (CCP4, Didcot, GBR); McNicholas, Stuart (YSBL, York, GBR); Thomas, Jens (Institute of Integrative Biology, Liverpool, GBR); Uski, Ville (CCP4, Didcot, GBR); Rigden, Daniel (Institute of Integrative Biology, Liverpool, GBR); Keegan, Ronan (CCP4, Didcot, GBR)

Performing molecular replacement (MR) with low resolution data presents many challenges. A plethora of new techniques are available to isolate fragment search models and perform post-MR density modification suitable for high resolution data, but many of these are not applicable where the data resolution is no better than 3 Angstroms. Here, we rely on the availability of search models constituting a sizeable fraction of the scattering content of the target crystal. The selection and preparation of such search models is key to the success of the MR procedure. The correct placement of a model in the unit cell of the target relies heavily on not just the sourcing of the search model, but also on the selection from the model of that part of it most similar to the target. This selection process can be guided by exploiting any available information such as sequence alignment or the alignment of homologues against similar homologues to isolate a common structural core. In difficult cases, a lot of trial and error can be required to optimize both the choice of model and its preparation. Automated MR pipelines such as MoRDa and MrBUMP have been developed to derive many potential search models automatically. They make use of leading structural bioinformatics techniques to identify candidate search models and help guide the selection of atoms from that model to optimize their similarity to the target. Here we present how these programs work, as well as how they can help in cases of low resolution structure solution. 